\title{
PROCESOS AUTOEVALUATIVOS ENTRE DOCENTES UNIVERSITARIOS: APROXIMACIÓN MEDIANTE CASOS EN EL CONTEXTO DE UNA INSTITUCIÓN PRIVADA
}

\author{
SELF-ASSESSMENT PROCESSES AMONG \\ UNIVERSITY PROFESSORS: APPROACH \\ THROUGH CASES IN THE CONTEXT OF
} A PRIVATE INSTITUTION

Luis Medina Velázquez Marco Antonio Rigo Lemini

Doctor en Evaluación Educativa, Universidad Anáhuac, México. Coordinador del Posgrado en Educación, Universidad Anáhuac, México. lmedina@anahuac.mx

Doctor en Psicología Educativa, Universidad Nacional Autónoma de México (UNAM). Docente e investigador universitario. Facultad de Psicología, Universidad Nacional Autónoma de México (UNAM).

marcoantoniorigo@prodigy.net.mx 


\section{RESUMEN}

Este trabajo explora los procesos de autoevaluación y autorregulación de la práctica docente entre cuatro profesores universitarios. $\mathrm{Su}$ objetivo es identificar los mecanismos a través de los cuales estos profesionales analizan y valoran su propio desempeño, así como las estrategias que despliegan para mejorarlo. La investigación examina las creencias y los saberes de los enseñantes acerca de la evaluación institucional del desempeño docente, y sus implicaciones sobre los fenómenos de autorregulación y toma de decisiones. El abordaje se ampara en el paradigma mediacional centrado en el pensamiento del maestro y desarrolla un estudio de casos con personal académico participante en un programa de Maestría en Educación. Se constató que los profesores enfrentan una amplia gama de situaciones y problemas frente a los cuales practican una toma de decisiones compleja y apremiante, que no siempre conduce a la optimización de su quehacer cotidiano.

Palabras clave: educación superior, evaluación docente, autorregulación docente, estudio mediante casos, toma de conciencia didáctica entre profesores.

\section{ABSTRACT}

This work explores the processes of self-evaluation and selfregulation of teaching practice among four university professors. We have been interested in identifying the mechanisms through which these professionals analyze and value their performance as well as the strategies they deploy trying to improve it. The research examines the beliefs and knowledge teachers have about the institutional evaluation of teacher performance and its implications on the phenomena of selfregulation and decision-making. The approach is based on the mediation paradigm focused on teacher thinking and develops a case study with academic staff participating in a Master's in Education program. It was found that teachers face a wide range of situations and problems against 
which they practice complex and pressing decision-making, which does not always lead to the optimization of their daily work.

Keywords: higher education, teacher evaluation, self-regulation in teachers, study of cases, didactic awareness in professors.

\section{INTRODUCCIÓN}

El acercamiento a la temática docente es siempre complejo e intrincado, multidimensional y multifactorial, a la vez que necesario y en este momento más bien apremiante. Existen diversas aristas que han de considerarse: la selección y el reclutamiento de los enseñantes, su formación y actualización continua, la práctica de la enseñanza y la necesaria reflexión en torno a ella, la participación de maestros en los procesos de diseño, desarrollo e interpretación curricular, así como la evaluación del saber, el pensamiento y las prácticas magisteriales. En el presente documento, nos concentraremos en este último aspecto. En particular, analizaremos las posibilidades y los recursos que los educadores formales se encuentran en posibilidades de desplegar cuando se valoran a sí mismos con la intención de perfeccionar su propio desempeño.

De acuerdo con Atkinson y Claxton (2008), en el proceso de autoevaluación que llevan a cabo los profesores acerca de su práctica docente, la razón, sensibilidad e intuición son herramientas valiosas para examinar y reflexionar los resultados de su actuación. Como profesionales reflexivos, toman decisiones, emiten juicios, forman creencias y generan rutinas propias que contribuyen a su desarrollo en tanto enseñantes. Pero, ¿realmente son sus pensamientos los que primordialmente guían y orientan su conducta en el aula? ¿Podemos afirmar que existe una coherencia entre cogniciones y actuación, entre lo que se sabe y se cree, por una parte, y lo que se hace y se expresa, por la otra?

Lucio (2006) sostiene que es en el paradigma del enfoque reflexivo sobre la práctica donde se intenta comprender e interpretar a los profesores, dentro del marco de los problemas complejos que enfrentan en 
la escuela y la manera en que desarrollan sus capacidades para resolverlos. Así mismo, considera que este paradigma surge como una reacción a la racionalidad técnica del currículum y a la concepción positivista sobre la ciencia, en un intento por superar la relación lineal y mecánica del conocimiento científico-técnico y la práctica del profesorado en las aulas.

De ahí que muchos estudios se han centrado en conocer cómo se traducen las creencias del profesor, su concepción sobre la enseñanza, las teorías implícitas y los constructos personales que posee en su labor dentro del aula; y a su vez, determinar cómo repercuten sus vivencias, acciones y experiencias didácticas, sobre la conformación de su manera de entender y concebir la docencia.

Este trabajo de investigación examinó la manera en que tiene lugar la autorregulación de la práctica docente al interior de un grupo de profesores universitarios, así como reconocer los factores que influyen en esta. Es decir, su propósito fue analizar las modificaciones que los profesores imprimen a sus procesos de enseñanza, con base en el autoanálisis de su actuación y pensamiento didáctico, la atribución de resultados de su evaluación docente y los procesos de reflexión que llevan a cabo. Lo anterior implicó un proceso de indagación caracterizado por el examen sistemático y en profundidad de casos de entidades sociales o educativas únicas. Es decir, a través del estudio de la particularidad y de la complejidad de casos singulares, en un intento de conocer cómo funcionan todas las partes que los componen y las relaciones entre ellas para formar un todo (Stake, 2006).

En este sentido, los objetivos pretendidos fueron:

a. Identificar saberes y creencias de los profesores acerca de la evaluación institucional del desempeño docente a la que son sujetos.

b. Examinar las formas de proceder de los profesores universitarios en las tareas de autoevaluación y atribución de sus propios resultados de evaluación magisterial. 
c. Caracterizar las formas de proceder de los profesores universitarios en los procesos de reflexión crítica y autorregulación docente que llevan a cabo.

d. Analizar los diferentes factores que inciden en las decisiones que el profesor toma para mejorar su desempeño docente.

e. Constatar si la información que reciben los profesores, a través de la evaluación docente, se traduce realmente en acciones de mejora.

\section{CONTEXTO INSTITUCIONAL}

La institución objeto de estudio es una universidad privada de educación superior de inspiración católica, fundada en 1964; forma parte de una red con presencia nacional e internacional. Su sistema educativo se edifica sobre los más altos valores y concepciones del hombre, de la sociedad y la vida: el respeto a la dignidad de la persona, una visión profundamente humana y social de la profesión, un convencimiento profundo de que la razón es capaz de conocer la realidad y una sólida formación moral. Los docentes que participaron en el estudio colaboran en el Posgrado de la Facultad de Educación, en un programa de Maestría con 30 años de existencia, reacreditado con nivel uno por los Comités Interinstitucionales para la Educación Superior.

La evaluación institucional de maestros, por su parte, se efectúa a través de varios mecanismos. El principal, y al que aludiremos centralmente en el presente trabajo, es un cuestionario aplicado masivamente a los estudiantes, en donde valoran a sus profesores al término de cada curso de acuerdo a las siguientes categorías:

- Planeación: refleja (en dos reactivos) el grado de cumplimiento del programa y si el profesor lo dio a conocer o no al inicio del curso.

- Habilidades y estrategias didácticas: engloba (en seis reactivos) las actividades que desarrolla el profesor para facilitar y asegurar el aprendizaje. 
- Evaluación del aprendizaje: busca valorar (en tres reactivos) la congruencia, justicia y oportunidad con que el docente evalúa el desempeño del alumno.

- Rasgos profesionales y personales: intenta sopesar (a través de cinco reactivos) los atributos de personalidad y características del maestro como profesionista.

- Identificación institucional: aquilata (a través de dos reactivos) el sentido ético y la integración del profesor en la universidad, así como sus contribuciones a la formación integral del alumno.

- Valoración general del curso: pretende que el alumno lleve a cabo una apreciación general acerca del desarrollo del curso, la carga de trabajo implicada en este, así como los resultados de la asignatura (incorpora tres reactivos).

Los resultados obtenidos a través de la aplicación de este instrumento - cuestionables desde el punto de vista de algunos de los docentes evaluados-, constituyen un punto de partida irrenunciable para la toma de decisiones relacionadas con la asignación a grupos o espacios curriculares, así como el diseño y desarrollo de actividades formativas orientadas a la mejora del trabajo magisterial.

\section{SUSTENTO TEÓRICO}

Como afirma Álvaro Marchesi (cit. en Vélaz de Medrano y Vaillant, 2011: 7): «La calidad de la educación de un país no es superior a la calidad de su profesorado. De ahí la prioridad que la gran mayoría de las reformas educativas otorgan al fortalecimiento de la profesión docente». En otras palabras, al ser una figura central en la calidad educativa, el docente ha constituido una fuente de estudio y reflexión importante en el contexto pedagógico.

A este respecto es importante destacar, de acuerdo con Monereo et al. (1997), que la enseñanza es una actividad sumamente compleja desarrollada en circunstancias particulares y difíciles de prever. Por tanto, 
resulta esencial ofrecer a los profesores instrumentos de interpretación y análisis de la situación en que desarrollan su quehacer pedagógico para decidir respecto a su actuación como aprendices y docentes estratégicos, de manera que se vaya enriqueciendo y ampliando su formación en la interacción con la realidad cotidiana de la práctica profesional.

Con base en lo anterior, una de las principales preocupaciones que en los últimos años ha captado la atención de numerosos profesionales de la educación superior, se relaciona con comprender el pensamiento reflexivo y metacognitivo del profesor. El enfoque metacognitivo propiamente dicho - derivado de las investigaciones en Psicología Cognitiva- se refiere al grado de conciencia o conocimiento que los individuos poseen sobre su forma de pensar (procesos y productos cognitivos). Es decir, la forma que tienen los maestros de tomar conciencia de sus procesos de pensamiento y actuación docente, y de reflexionar crítica y sistemáticamente sobre estos; pero también de contar con las estrategias y la disposición requeridas para transformarlos, reelaborarlos o reconstruirlos con la intención de mejorar.

La investigación se inspira en buena medida en el modelo educativo centrado en el pensamiento del maestro (Díaz Barriga y Rigo, 2003), el cual parte del supuesto de que la enseñanza puede considerarse como una dinámica de planificación y ejecución de actuaciones, un proceso de adopción de decisiones basadas -implícita o explícitamente- en las creencias, la intuición, la reflexión crítica y los conocimientos profesorales.

Por otra parte, recupera los planteamientos de diversos autores como Dewey (cit. en Navarro, Illesca y Lagos, 2005), Schön (1998), Monereo et al. (2009) y Hugo (2008), acerca del pensamiento reflexivo, estratégico y emocional del profesor, para estudiar la dinámica y paradójica relación entre dichos aspectos en el proceso de autorregulación de la práctica docente. De acuerdo con estos autores, las dificultades o resistencias que pueden experimentar los profesores para transformar sus prácticas de forma sostenida se relacionan con aspectos tales como: sus concepciones sobre la enseñanza; su identidad docente; la etapa de desarrollo y consolidación en la que se encuentran en su carrera profesional; el contexto institucional en el que colaboran; el tipo 
de asignaturas que imparten; el grado de autoconocimiento que tienen de sí mismos como docentes; el desgaste emocional y los sentimientos de inseguridad, asociados a la necesidad del cambio y la mejora; los compromisos que implica ponerlos en práctica, entre otros.

Además del binomio «razón-emoción», la investigación se apoya en lo que señalan otros autores acerca de la importancia de la naturaleza intuitiva de la labor docente, que se vive en el momento mismo de la clase y que es casi imposible de prever. Atkinson y Claxton (2008) la describen como un medio de conocimiento privilegiado y enigmático, que implica cierta sensibilidad, capacidad de juicio, pericia y cavilación. A pesar de que la intuición goza de mala reputación, debido a su naturaleza subjetiva (que aparentemente la aleja de la «capacidad de ser racional e infalible»), es una manera fluida y flexible de conocer o comprender situaciones nuevas y complejas, cuando no se está en capacidad de teorizar a partir de la propia pericia, pues permite realizar juicios sutiles y con cierta precisión basados en la experiencia. Se asume igualmente el enfoque de las teorías implícitas, en el sentido de identificar la consistencia de las distintas concepciones tácitas acerca del proceso de enseñanza-aprendizaje, la propia evaluación y autorregulación docentes (Pozo et al., 2006).

En lo que respecta a la evaluación de la docencia, conviene señalar que para Loredo et al. (2000) esta práctica en la educación superior mexicana cuenta su historia en solo unas décadas, y a pesar de ser reciente, evoluciona con rapidez. Sin embargo, tradicionalmente ha venido cumpliendo una función vinculada a la administración y el control, por lo que es considerada por muchos profesores como práctica ineficaz y poco satisfactoria. También reconoce que la evaluación docente que toma en cuenta la opinión de los estudiantes, ofrece una aproximación al desempeño del profesor en el aula. Es decir, informa acerca del desarrollo del curso y las actividades de enseñanza-aprendizaje, el clima del aula y la satisfacción del alumno, el grado de compromiso y cumplimiento del enseñante, entre otras cosas.

Para comprender mejor las experiencias institucionales, relacionadas con la evaluación docente, es importante mencionar que un estudio realizado por Rueda, Elizalde y Torquemada (cit. en Elizalde y 
Reyes, 2008), advierte que en las universidades mexicanas es común la existencia generalizada de prácticas de evaluación profesoral caracterizadas por poseer una concepción, antigüedad y uso de los resultados claramente diferenciados. En algunas instituciones, la evaluación es una práctica aceptada y forma parte de la vida académica, mientras que en otras es un requisito administrativo, generalmente concebido como condición para acceder a una compensación salarial.

En términos generales, entre los agentes que impulsan la evaluación magisterial en las distintas universidades, se aprecia la presencia de autoridades, coordinaciones y planes institucionales, encargados del desarrollo de la docencia y la evaluación. En todos los casos se declaró, por parte de los entrevistados, que con la realización de las tareas evaluativas persiguen objetivos tanto académicos como institucionales, entre los cuales destacan la retroalimentación, la mejora en la calidad de la docencia, la planeación y la sensibilización de los enseñantes sobre la importancia de su propia evaluación.

\section{ESTRATEGIA METODOLÓGICA}

\section{a. Elementos generales}

Se trata de una investigación mixta, de corte predominantemente cualitativo, de carácter descriptivo e interpretativo, en la que se asumen algunos principios de la metodología fenomenográfica en el sentido de indagar explícitamente cómo los docentes viven o interpretan los procesos de enseñanza-aprendizaje y las experiencias de evaluación docente en que participan.

El presente trabajo se apoya en el estudio de casos en tanto que, de acuerdo con Pérez Serrano (1994), busca llevar a cabo una descripción intensiva y holística, así como el análisis del complejo fenómeno social antes descrito, basándose en el razonamiento inductivo al manejar múltiples fuentes de datos. Mediante dicho método se recogen, de forma descriptiva, distintos tipos de informaciones cualitativas que no 
aparecen reflejadas en números sino en palabras. Lo esencial en esta metodología es poner de relieve incidentes clave mediante el empleo de entrevistas realizadas a profesores (sobre autoeficacia, atribución de los resultados de su evaluación docente, etcétera) y el análisis de documentos o producciones elaboradas por estos (sus programas, materiales instruccionales, etcétera), que de alguna manera reflejan los ajustes que llevan a cabo en su quehacer educativo cada vez que imparten la misma asignatura a un grupo distinto, después de haber sido evaluados. El fin último de ello es tratar de iluminar la comprensión del objeto de estudio: sus procesos de autoevaluación y autorregulación docente.

\section{b. Preguntas de investigación}

Dada la complejidad del trabajo, podemos agruparlas en cuatro grandes categorías:

- b.1. Identidad, labor educativa y contex to en que se desenvuelve el enseñante: ¿Quién es este profesor?, ¿qué rasgos personales y profesionales distinguen su perfil como profesor?, ¿qué características generales identifican su práctica docente?, ¿en dónde, para quién y bajo qué circunstancias actúa?, ¿qué enseña y desde cuándo?, ¿qué hace para enseñar, para qué y cómo lo hace?, ¿cómo vive su labor docente?

- b.2. Pensamiento didáctico y práctica docente. ¿Qué piensa acerca de la docencia y cómo lo manifiesta?, ¿cuáles son sus principales concepciones y preocupaciones pedagógicas?, ¿qué piensa acerca de lo que significa ser profesor universitario?, ¿cuáles son los aspectos que a su juicio caracterizan a un buen profesor universitario?, ¿qué características describen su práctica docente?, ¿qué percepción tiene de su eficacia docente?

- b.3. Punto de vista sobre la evaluación institucional del desempeño docente y la atribución que hace de los resultados que recibe acerca de esta. ¿Qué opina acerca de la evaluación docente de la que es objeto?, ¿cómo se entera, interpreta y vive los resultados de su evaluación docente?, ¿qué impacto emocional y cognitivo ha tenido en él?, ¿a qué le atribuye los resultados?, ¿qué hace y qué otros referentes toma en 
cuenta para analizarla más a fondo?, ¿a qué aspectos de la evaluación docente les otorga más valor?

- b.4. Su experiencia con respecto a los procesos de autoevaluación y autorregulación que lleva a cabo. ¿Qué tanto se vincula en procesos de autoevaluación, mejora y toma de decisiones?, ¿cómo y con qué criterios se autoevalúa?, ¿a qué criterios les otorga más valor?, ¿qué clase de decisiones toma a partir de esa autoevaluación?, ¿en qué medida la información que recibe, a través de la evaluación docente, se traduce en su participación en actividades de mejora, tales como participación en cursos, lecturas, etcétera?, ¿qué tipo de decisiones didácticas derivan de su participación en estas actividades?, ¿cómo se reflejan las decisiones didácticas — resultado de sus procesos de autoevaluación y autorregulación- en su desempeño como profesor y en los propios resultados de la evaluación docente?

\section{c. Participantes}

Como lo sostiene Stake (2000), la investigación con estudio de casos no tiene el interés de lograr representatividad o de establecer generalizaciones. El propósito primordial del estudio de casos — dada su naturaleza única y compleja-, es la comprensión de los otros en sus diversas interrelaciones dentro de su escenario cultural.

Para la confección de la muestra, se eligieron cuatro docentes con características disímiles en cuanto a los siguientes aspectos:

- Por su tipo de relación laboral con la universidad: dos profesores cobran por honorarios y dos son de planta.

- Por su género: una mujer y tres hombres.

- Por el tipo de licenciaturas de origen: un psicólogo, un comunicólogo y dos pedagogos.

- Por los grados académicos: dos maestros y dos doctores. 
- Por su trayectoria laboral y profesional: profesionistas de corta, mediana y larga trayectoria.

- Por su experiencia y formación docente: algunos profesores con estudios de licenciatura asociados al campo educativo y otros con estudios vinculados al ámbito organizacional.

- Por los campos disciplinares en los que centran su docencia: dos se enfocan al ámbito organizacional, uno al campo pedagógicocurricular y otro al de tecnología educativa.

\section{d. Escenarios}

Como se ha mencionado, la investigación fue realizada en una institución privada de educación superior con acreditado prestigio y ubicada dentro del área metropolitana de la Ciudad de México. Los espacios específicos donde se llevó a cabo el levantamiento de datos fueron, en el caso de los profesores de planta, las oficinas administrativas de la Facultad o Escuela a la que se encuentran adscritos y, en el caso de los profesores que cobran por honorarios, el salón de maestros destinados a dichos profesores. Consideramos que dichos espacios contaron con las dimensiones, la accesibilidad, el mobiliario, la ventilación e iluminación, las condiciones térmicas, así como de aislamiento de ruido y de distractores externos, necesarios para el adecuado levantamiento de datos.

\section{e. Procedimiento}

Con el propósito de realizar el levantamiento de información, se emplearon diversas técnicas de investigación: entrevista estructurada de los actores que conformaron los cuatro casos de estudio; aplicación auto-administrada de escalas de evaluación docente; análisis de evidencias del trabajo de los profesores, conformadas por sus programas magisteriales, materiales instruccionales y materiales evaluativos; así como el análisis descriptivo de los resultados institucionales de evaluación del desempeño docente de cada profesor. 
- e.1. La entrevista consideró varias categorías relacionadas a las características de los profesores, su pensamiento docente, su autoevaluación docente y evocación de sus resultados institucionales, sus procesos de atribución de resultados de evaluación docente y de autorregulación de la práctica.

- e.2. La aplicación de escalas auto-administradas implicó la presentación, por parte del investigador, de los instrumentos de evaluación docente a los actores de cada caso de estudio, para que los contestaran de manera autónoma. Así mismo, dado que los instrumentos fueron contestados en presencia del investigador, este estuvo al pendiente de resolver las dudas que pudieran presentarse al respecto del llenado del documento.

- e.3. Para llevar a cabo el análisis de evidencias de las producciones docentes, el investigador se implicó en la lectura crítica y analítica, así como en la interpretación de los programas, materiales instruccionales y evaluativos diseñados por cada profesor, con el fin de identificar las distintas concepciones implícitas sobre el proceso de enseñanza-aprendizaje.

- e.4. Finalmente, el análisis de los resultados institucionales de evaluación del desempeño docente de cada profesor se llevó a cabo desde un enfoque descriptivo. En primera instancia, acerca de los promedios numéricos obtenidos en distintas dimensiones del desempeño docente que fueron valoradas por los estudiantes. Y en segundo lugar, acerca de las fortalezas y áreas de oportunidad identificadas a través de los comentarios y observaciones de dichos estudiantes.

\section{f. Instrumentos para la recogida de datos}

Como se desprende del apartado anterior, fueron muy diversas las técnicas y actividades efectuadas para la recogida de datos. A fin de presentarlas simplificadamente, en la tabla 1 se consignan los principales instrumentos empleados, así como las dimensiones fundamentales que cada uno de ellos estaba orientado a explorar. 


\section{Tabla 1. Instrumentos utilizados a lo largo de la investigación}

\begin{tabular}{|c|c|c|c|c|c|}
\hline & \multicolumn{4}{|c|}{ Núcleos de interés } \\
\hline & & $\begin{array}{l}\text { Identidad del } \\
\text { profesor, su labor } \\
\text { educativa y el } \\
\text { contexto en el que } \\
\text { se desenvuelve }\end{array}$ & $\begin{array}{c}\text { Pensamiento } \\
\text { didáctico y } \\
\text { práctica docente }\end{array}$ & $\begin{array}{c}\text { Percepción de la } \\
\text { evaluación } \\
\text { institucional del } \\
\text { desempeño } \\
\text { docente y atribución } \\
\text { que hace de los } \\
\text { resultados }\end{array}$ & $\begin{array}{c}\text { Procesos de } \\
\text { autoevaluación y } \\
\text { autorregulación } \\
\text { docente que lleva a } \\
\text { cabo el profesor }\end{array}$ \\
\hline \multirow{8}{*}{ 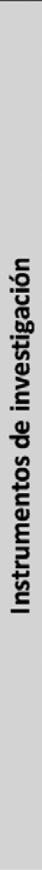 } & \begin{tabular}{|l|}
$\begin{array}{l}\text { 1. Guía de entrevista sobre } \\
\text { pensamiento, acción, autoestima } \\
\text { y autoconcepto profesional }\end{array}$ \\
\end{tabular} & & & & \\
\hline & $\begin{array}{l}\text { 2. Escala para analizar las } \\
\text { modificaciones hechas por el } \\
\text { profesor a su programa } \\
\text { magisterial }\end{array}$ & & & & \\
\hline & $\begin{array}{l}\text { 3. Escala para analizar las } \\
\text { modificaciones hechas por el } \\
\text { profesor a sus materiales } \\
\text { instruccionales }\end{array}$ & & & & \\
\hline & $\begin{array}{l}\text { 4. Escala para analizar las } \\
\text { modificaciones hechas por el } \\
\text { profesor a sus materiales } \\
\text { evaluativos }\end{array}$ & & & & \\
\hline & $\begin{array}{l}\text { 5. Cuestionario sobre } \\
\text { autoeficacia docente del } \\
\text { profesor universitario }\end{array}$ & & & & \\
\hline & $\begin{array}{l}\text { 6. Guía de entrevista sobre el } \\
\text { proceso de atribución de los } \\
\text { resultados de evaluación } \\
\text { docente, autoevaluación y } \\
\text { autorregulación }\end{array}$ & & & & \\
\hline & $\begin{array}{l}\text { 7. Escala de evaluación docente } \\
\text { para el análisis de evocación de } \\
\text { resultados }\end{array}$ & & & & \\
\hline & $\begin{array}{l}\text { 8. Escala de evaluación docente } \\
\text { para el análisis la autoevalución } \\
\text { del profesor }\end{array}$ & & & & \\
\hline
\end{tabular}

\section{ANÁLISIS DE RESULTADOS}

En general, los cuatro profesores comparten una concepción humanista del quehacer educativo, al considerar que se orienta al desarrollo integral de las capacidades de la persona y que ello posee gran trascendencia social. El profesor I manifiesta de forma explícita una visión artística de esta labor y una profunda preocupación por satisfacer las 
expectativas del alumno, y sostiene que le satisface más el trabajo didáctico. El profesor II se siente orgulloso por la contribución social de su trabajo pues, además de la docencia, le apasiona colaborar en procesos de actualización docente; en tanto que el III y el IV enfatizan el carácter valoral del proceso educativo. Al más joven de estos dos, le complace mucho su trabajo como investigador, en tanto al otro, su labor como empresario y coach ejecutivo.

A través de este trabajo de investigación se constató que en los cuatro casos de estudio, el quehacer profesional de los docentes efectivamente se caracteriza por su complejidad y dinamismo, ya que ocurre en un contexto multidimensional y continuamente cambiante. Todos coinciden al señalar la pertinencia, interdisciplinariedad, flexibilidad y actualización del plan de estudios y enfatizan que, por un lado, existe cierta riqueza al contar con estudiantes que provienen de diversidad de campos formativos, al tiempo que por el otro, muchos no cuentan con formación o experiencia tecno-pedagógica o de habilidades de aprendizaje estratégico.

Las dificultades y los problemas que reportan se han clasificado de dos maneras: las de carácter endógeno (atribuibles al propio profesor y, por tanto, que dependen en buena medida de las acciones que emprenda para regularlas); o bien exógenas (no atribuibles al docente $\mathrm{y}$, por lo tanto, que obedecen en gran parte a las circunstancias del contexto institucional y social). Entre los aspectos endógenos, caben señalar los relacionados con:

- Formación y experiencia en el campo disciplinar en el que enseñan.

- Formación y experiencia pedagógica.

- Pensamiento didáctico-curricular.

- Rasgos personales (edad, sexo, personalidad, etcétera).

- Rasgos profesionales como docente (vocación, sentido de identidad institucional, compromiso con la calidad de su trabajo, etcétera). 
- Y entre los de carácter exógeno, aspectos relacionados con:

- Altas expectativas y demandas del alumno de posgrado.

- Gran heterogeneidad del perfil de los estudiantes (originada principalmente en función de sus licenciaturas de origen).

- Que los estudiantes no dediquen tiempo completo a sus estudios de maestría para poder trabajar.

- Que algunos no cuenten con experiencia laboral.

- Múltiples funciones académicas que desarrollan.

- Fuerte y cotidiana carga de trabajo.

- Altas expectativas y demandas de la institución, entre otras.

$\mathrm{Al}$ referirse al fin último de la docencia, el profesor I lo considera un arte, en que la didáctica cumple una función sustantiva para manejar asertivamente los conflictos emocionales y cognitivos del alumno. De esta manera, busca promover los valores y el pensamiento crítico del alumno. Se concibe como un didacta ecléctico, en tanto que afirma tomar lo mejor de cada paradigma considerado en la Psicología Educativa.

El profesor II enfatiza la importancia de la eficiencia técnica del quehacer docente. Se declara a favor del constructivismo, y se considera un docente activo y práctico. Sostiene que su principal objetivo es que el alumno sea un aprendiz autónomo.

El profesor III destaca su labor como guía. Afirma que su propósito principal es formar para la vida y los valores. Cree en el aprendizaje activo y conectado con la realidad. Se considera crítico e innovador y un promotor del trabajo en equipo.

Finalmente, el profesor IV se identifica a favor de los principios del constructivismo. Afirma que busca enseñar a pensar, cuestionar, 
profundizar y argumentar al alumno, así como a construir su pensamiento autónomo.

A este respecto, a través de la evaluación institucional, los estudiantes reconocen en los cuatro profesores un perfil altamente profesional y de gran calidad humana; dominio del contenido; entusiasmo, pasión y entrega en su desempeño; capacidad para motivar y mantener el interés del grupo; vinculación de las asignaturas con las necesidades actuales del mundo laboral, entre otros aspectos.

Los profesores reportan estar pendientes de los resultados de la evaluación docente, salvo uno. Los profesores I y II los consultan una vez que han terminado de subir las calificaciones finales de sus asignaturas al registro electrónico de la universidad y, posteriormente, los comentan con el coordinador del programa. Por su parte, el profesor IV prefiere revisar los resultados de su evaluación docente cada inicio del período escolar y busca el momento más oportuno en que su estado anímico le fortalezca para enterarse de estos. Sostiene que lo ideal es que la evaluación se lleve a cabo en un clima de confianza orientado a la mejora, más que a la rendición de cuentas. En contraste, el profesor III comenta que hasta el momento no ha ingresado a la intranet de la universidad para enterarse de sus resultados.

Los cuatro profesores reportan estar habituados a autoevaluarse a lo largo de todo el curso, sea de manera consciente o inconsciente. Al principio, para reconocer las características del grupo y las estrategias didácticas que emplearán en función de las reacciones y la conexión lograda con el grupo. En el día a día, examinan el desempeño del alumno y emplean como evidencia de ello los trabajos y exámenes parciales. Y al final, identifican cuáles fueron los principales aprendizajes del grupo, así como sus impresiones acerca del curso. Entre los principales motivos que los mueven a autoevaluarse se encuentran el compromiso con los alumnos y la calidad, el logro de objetivos personales, mantenerse actualizados y corregir sus prácticas educativas.

Para terminar conviene reconocer que, desde un punto de vista formativo, los resultados de evaluación permiten a los educadores participantes tomar conciencia acerca de sus aciertos y errores, así como 
reflexionar y emprender acciones para mejorar de manera continua. Desde un punto de vista emocional, cuando tales resultados han sido positivos se han sentido muy satisfechos al considerar que las autoridades académicas reconocen y aprecian el profesionalismo de su trabajo, además de fortalecer su autoestima como académicos universitarios. En contraste, cuando han sido desfavorables, les han creado la sensación de no haber cumplido con lo que se esperaba de ellos, experimentar desgaste emocional, frustración y hasta llegar a sentirse indignados, especialmente cuando las críticas que reciben se refieren no tanto a su desempeño docente, sino a su propia persona.

\section{CONCLUSIONES}

Resulta importante insistir en que, de acuerdo con Hernández y Sarramona (2002), la tarea de ser profesor universitario es muy compleja. En primer lugar, porque en la actualidad la educación superior se enfrenta en todo el mundo a múltiples desafíos y dificultades. Y en segundo término, porque se trata de una ocupación polivalente que demanda el dominio de diversas competencias. De modo que, si en otros tiempos era posible identificar al docente con la mera tarea de enseñar, en la actualidad su función comprende ámbitos de intervención mucho más amplios y vinculados a las demandas del entorno social.

Como quiera que sea, si se considera que la actividad sustantiva de este profesional es la enseñanza, puede afirmarse que es deber del maestro conocer a fondo el contenido de su materia y realizar suficientemente bien sus actividades docentes; pero sobre todo debe ser un reflejo de los valores que desee inculcar, reflejo tan nítido y brillante que motive a los demás a hacerlos propios. Coincidimos con Díaz Barriga (2005) en que las decisiones que los docentes realizan sobre su ejercicio didáctico son especialmente importantes; han de ser asumidas, de modo fundamentado y reflexivo, durante la planeación, instrumentación, implantación, evaluación, retroalimentación y los procesos de ajuste inherentes a su trabajo magisterial.

Asimismo, coincidimos con Rueda (2008) en que a pesar de que este vínculo entre los resultados evaluativos de todo tipo a que se encuentra 
expuesto el enseñante universitario y la dinámica de autoevaluación, reflexión y ajuste en y sobre sus tareas didácticas cotidianas puede parecer natural e inevitable, la realidad es que no siempre ocurre así. O no como esperaríamos. Y este divorcio se traduce en una eventual pérdida de sentido de las evaluaciones docentes, entre las que se encuentra la que deriva de la aplicación de cuestionarios de opinión entre los alumnos y la que se desprende del ejercicio valorativo de las autoridades institucionales en sus diferentes niveles jerárquicos.

Con base en lo anterior, es posible sostener que para detectar las necesidades de formación - tanto inicial como continua- de los profesores en ejercicio, es fundamental examinar dicha dinámica hetero y autoevaluativa para que, a partir de ella, se señale un conjunto de orientaciones que permitan efectuar las tareas sustentado en un modelo orientado a la autorregulación, que se inicia a nivel interpersonal y se vuelve intrapersonal a través de la reflexión crítica, la metacognición y los procesos motivacionales que median y promueven las decisiones.

Los cambios profesorales solo serán posibles si las personas directamente implicadas toman conciencia de la situación, reflexionan sobre la realidad y deciden pertinentemente para su transformación. Es así que, a partir de este trabajo, se espera generar nuevas vetas de investigación para estudiarse desde una perspectiva mixta, con el fin de producir conocimiento acerca del sentido y la significación de acciones y situaciones humanas, cuidando al mismo tiempo una relativa precisión en la medida y una progresiva generalización de los resultados.

Finalmente, creemos que esta investigación es tan solo un punto de partida del que se desprenden gran número de inquietudes y cuestionamientos, algunos de los cuales pueden encaminarse a profundizar en los siguientes aspectos:

- Análisis de los factores asociados al proceso que siguen los profesores para enterarse de los resultados de su evaluación docente; interpretar o leer la información que se les proporciona; afrontar asertivamente el impacto emocional y cognitivo que ello les provoca; examinar de forma más reflexiva dichos resultados para 
identificar alternativas o vías de acción, tomar decisiones e implicarse en la implementación de las mismas; monitorear dicha implementación; verificar los resultados obtenidos, etcétera.

- Identificación de los estilos de afrontamiento de que disponen los profesores para manejar asertivamente las emociones provocadas por los resultados de evaluación del desempeño docente a fin de implicarse en procesos de autorregulación y mejora, entendiendo como «afrontamiento» al conjunto de actividades que el individuo puede poner en marcha — tanto de tipo cognitivo como emocional- para manejar solventemente dicha situación.

- Desarrollo, aplicación y evaluación de procesos y actividades de formación docente inicial y continua que favorezcan el aprendizaje estratégico de los procesos de autoevaluación docente, la autorregulación del componente emocional y cognitivo, así como la toma de decisiones para emprender acciones de mejora.

\section{REFERENCIAS}

Atkinson, T. y Claxton, G. (2008). El profesor intuitivo. Barcelona: Octaedro.

Díaz Barriga, F. (2005). Enseñanza situada: vínculo entre la escuela y la vida. México: Mc Graw-Hill.

Díaz Barriga, F. y Rigo, M.A. (julio-septiembre, 2003). «Realidades y paradigmas de la función docente: Implicaciones sobre la evaluación magisterial en educación superior». Revista de la Educación Superior, vol. XXXII(3), n. 127, 53-61.

Elizalde, L. y Reyes, R. (2008). «Elementos clave para la evaluación del desempeño de los docentes». Revista Electrónica de Investigación Educativa, número especial. Recuperado de: http:/ / redie.uabc.mx/NumEsp1/contenido-elizaldereyes.html 
Hernández, M. y Sarramona, J. (2002). «La autoevaluación docente. Una propuesta para la formación profesional». Revista Bordón, n. 52, vol. 4, 559-576.

Hugo, D. (2008). Análisis del proceso de autorregulación de las prácticas docentes de futuras profesoras de ciencias, focalizado en sus emociones. Barcelona: Universidad Autónoma de Barcelona.

Loredo, J. et al. (2000). Evaluación de la práctica docente en educación superior. México: Editorial Porrúa-Universidad Anáhuac.

Lucio, R. (2006). «Algunos paradigmas de formación del profesorado y la Reflexión Metacognitiva. Un paso más hacia la concreción del Enfoque Reflexivo-Crítico». Revista Docencia, n. 29, Colegio de profesores de Chile, 79-89.

Monereo, C. (coord.), Castello, M., Clariana, M., Palma, M. y Pérez, M.L. (1997). Estrategias de enseñanza y aprendizaje. Formación del profesorado y aplicación en la escuela. Barcelona: Graó.

Monereo, C., Badia, A., Bilbao, G., Cerrato, M. y Weise C. (2009). «Ser un docente estratégico: Cuando cambiar la estrategia no basta». Cultura y Educación, n. 21 (3), 237-256.

Navarro, N., Illesca, M. y Lagos, X. (2005). «Modelos de formación docente base para una innovación curricular». Revista de Educación en Ciencias de la Salud, vol. 2, n. 2, 93-96.

Pérez Serrano, G. (1994). Investigación cualitativa. Retos, interrogantes y métodos. Madrid: La Muralla.

Pozo, J.I., Scheuer, N., Pérez, M.P., Mateos, M., Martín, E. y De la Cruz, M. (2006). Nuevas formas de pensar la enseñanza y el aprendizaje. Las concepciones de profesores y alumnos. Barcelona: Graó.

Rueda, M. (2008). «La evaluación del desempeño docente en la universidad». Revista Electrónica de Investigación Educativa, Especial. Recuperado de: http:// redie.uabc.mx/NumEsp1/ contenido-rueda.html 
Schön, D. (1998). El profesional reflexivo. Cómo piensan los profesionales cuando actúan. Madrid: Paidós Ibérica.

Stake, R.E. (2000). Investigación con estudio de casos. Madrid: Morata.

Stake, R.E. (2006). Evaluación comprensiva y evaluación basada en estándares. Barcelona: Graó.

Vélaz de Medrano, C. y Vaillant, D. (coords.) (2011). «Metas Educativas 2021». Profesión docente. Aprendizaje y desarrollo profesional docente. Madrid: Santillana. 